\title{
Separable Subsurface Scattering
}

\author{
Jorge Jimenez ${ }^{1,2}$, Károly Zsolnai ${ }^{3}$, Adrian Jarabo ${ }^{1}$, Christian Freude $^{3}$, Thomas Auzinger $^{3}$, \\ Xian-Chun $\mathrm{Wu}^{2}$, Javier von der Pahlen ${ }^{2}$, Michael Wimmer ${ }^{3}$ and Diego Gutierrez ${ }^{1}$ \\ ${ }^{1}$ Universidad de Zaragoza $\quad{ }^{2}$ Activision-Blizzard $\quad{ }^{3}$ Vienna University of Technology
}
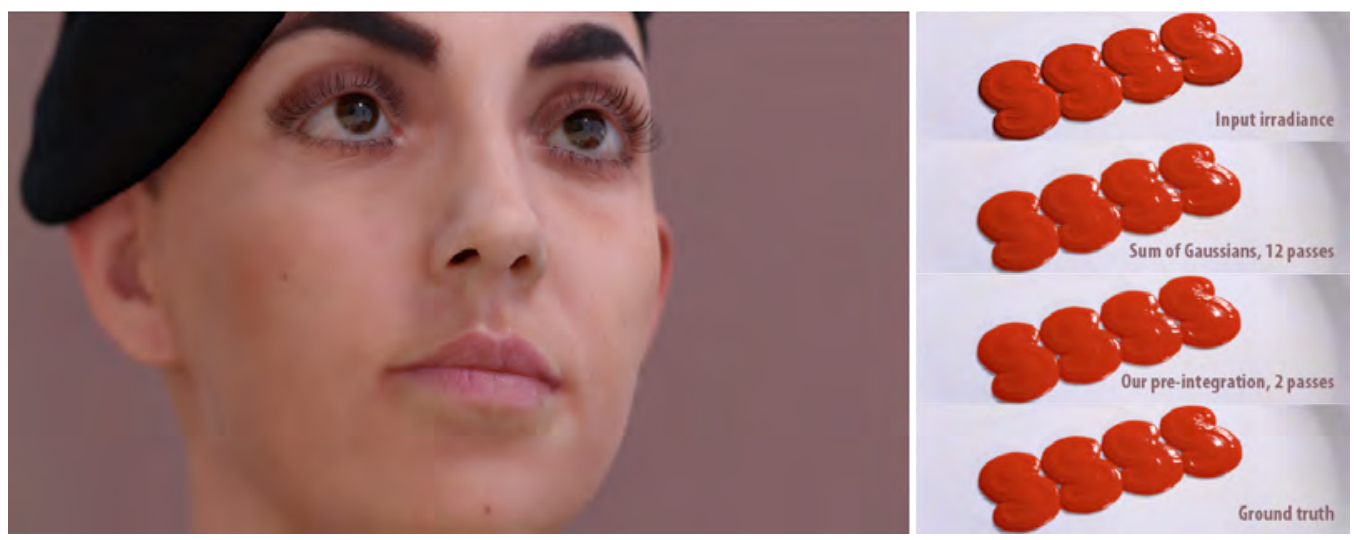

Figure 1: Real-time results of our method for simulating translucent materials (skin on the left, ketchup on the right). Our separable subsurface-scattering method enables the generation of these images using only two convolutions (versus 12 in the sum-of-Gaussians approach [dLE07, JSG09]) and seven samples per pixel, while featuring quality comparable with the current state of the art, at a fraction of its cost. It can be implemented as a post-processing step and takes only $0.489 \mathrm{~ms}$ per frame on an AMD Radeon HD 7970 at 1080p, which makes it highly suitable for challenging real-time scenarios.

\begin{abstract}
In this paper we propose two real-time models for simulating subsurface scattering for a large variety of translucent materials, which need under 0.5 milliseconds per frame to execute. This makes them a practical option for realtime production scenarios. Current state-of-the-art, real-time approaches simulate subsurface light transport by approximating the radially symmetric non-separable diffusion kernel with a sum of separable Gaussians, which requires multiple (up to twelve) $1 D$ convolutions. In this work we relax the requirement of radial symmetry to approximate a $2 D$ diffuse reflectance profile by a single separable kernel.

We first show that low-rank approximations based on matrix factorization outperform previous approaches, but they still need several passes to get good results. To solve this, we present two different separable models: the first one yields a high-quality diffusion simulation, while the second one offers an attractive trade-off between physical accuracy and artistic control. Both allow rendering subsurface scattering using only two $1 D$ convolutions, reducing both execution time and memory consumption, while delivering results comparable to techniques with higher cost. Using our importance-sampling and jittering strategies, only seven samples per pixel are required. Our methods can be implemented as simple post-processing steps without intrusive changes to existing rendering pipelines.
\end{abstract}

\section{Introduction}

The accurate depiction of translucent materials is an important but challenging topic in the motion picture and video game industries. Rendering realistic subsurface scattering
(SSS) implies simulating how light travels and scatters inside translucent media, which is an expensive process. While offline rendering scenarios can afford longer computation times, real-time applications, such as video games, impose severe 


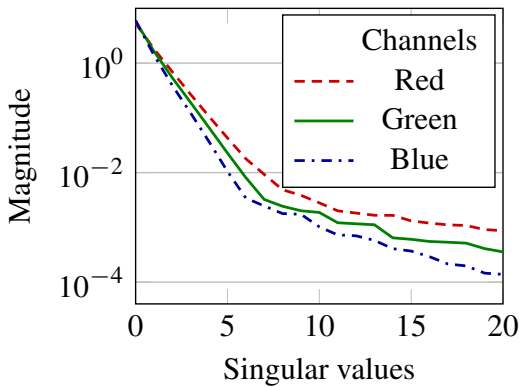

Figure 2: Decay of the singular values in the singular value decomposition of a diffuse reflectance profile used to simulate subsurface scattering in skin. Only the components associated to the first few singular values contribute appreciably to the reconstruction of the profile, making a low-rank approximation feasible.

time constraints, often leading to the exclusion of subsurface scattering and translucency effects. This in turn hinders the level of realism that can be achieved.

One of the most common approaches to compute subsurface scattering efficiently exploits the fact that it blurs highfrequency details and illumination. This means that simulating subsurface scattering can be approximated as a convolution with a diffusion kernel that mimics the diffuse reflectance profile for a given translucent medium. While the exact reconstruction normally requires an expensive two-dimensional convolution, d'Eon et al. [dLE07] showed that it can be approximated by a sum of radially symmetric Gaussians. Thus, due to the separability of Gaussians, the 2D convolution can be computed using a set of cheaper 1D passes, which allows high-quality skin rendering in real time. This approach was later extended to screen space, modulating the width of the kernel according to per-pixel depth information [JSG09].

However, in order to get adequate results, several Gaussians are needed to model the diffuse reflectance profile. This translates into multiple 1D convolutions per frame, which is still costly. In this paper, we make the key observation that exact simulated diffusion kernels, which are in general mathematically non-separable, can be closely reconstructed by a low-rank factorization for a wide range of materials (see Figure 2). Based on this, we present two different separable models that allow simulating subsurface scattering with just two 1D convolutions: The first one allows reconstructing a high-quality diffusion profile based on the observation that the irradiance is close to be additively separable, while the second is an artist-friendly model that, following the previous observation, provides an attractive trade-off between physical accuracy and ease of use for artistic editing of the scattering profiles (Table 1). Coupled with our importance-sampling and jittering strategies, our methods only require seven samples per pixel (see Figures 1 and 13).

\begin{tabular}{lccc}
\hline \multicolumn{1}{c}{ Model } & Visual quality & 1D convolutions & Separable \\
\hline 1 Gaussian & low & 2 & $\checkmark$ \\
2+ Gaussians & high & 2 per Gaussian & $x$ \\
\hline Kernel pre-integration & high & 2 & $\checkmark$ \\
Artist-friendly model & controllable & 2 & $\checkmark$ \\
\hline
\end{tabular}

Table 1: Compared to the state of the art [dLE07, JSG09] (above the separation line), our proposed techniques (below) offer solutions for a variety of trade-off choices. Our techniques are able to provide high-quality results by using a separable approximation of only two 1D convolutions.

Our methods can be implemented as simple postprocessing steps and do not rely on complex alpha-blending pipelines or Gaussian levels of detail [JG10], and work with dynamic objects without any additional cost. Moreover, all our rank-1 approximations execute in less then $0.5 \mathrm{~ms}$ per frame on modern commodity hardware and exhibit negligible fixed costs, as regions of the scene with no visible scattering can be quickly culled using stencil buffering. Our separable approximation of subsurface scattering fills the gap between physically based subsurface-scattering rendering and highly time-constrained environments such as games, and it is currently being used in game engines and production pipelines.

\section{Previous Work}

Offline techniques. The simulation of scattering inside translucent materials dates back to the radiative transfer equation [Cha60], which can be solved by traditional pathsampling techniques. The solution of this integral is a very demanding process in terms of computation time, especially if solved for a high number of bounces. Optimization techniques to reduce the computation times include using a dipole model [JMLH01, JB02] and modeling multiple scattering as a diffusion process [Sta95]. Donner and Jensen [DJ05] extended the dipole into a multipole model that allows modeling multi-layered translucent materials, such as skin. The same authors later introduced a photon diffusion technique to combine photon tracing and the diffusion approximation [DJ07] These works yield impressive results with computation times in the order of seconds per image. A more recent technique improves upon the inherent inaccuracies of the diffusion theory by using a more refined diffusion model to separate single and multiple scattering terms, alongside with the quantization of the Green's function of the diffusion equation to obtain realistic all-frequency results [DI11]. Further improvements revolve around better importance sampling [KF12], while a recent class of techniques rely on solving the searchlight problem by means of Monte Carlo integration imbued by multiple importance sampling [HCJ13].

Real-time techniques. Borshukov and Lewis [BL03] approximate subsurface scattering by blurring a 2D diffuse irradiance texture using a Gaussian filter. While this technique is 
efficient and maps well to the GPU, it neglects the more subtle details of subsurface scattering. This idea is later extended by d'Eon et al. [dLE07, dL07] to develop a high-quality realtime skin shader. They approximate the multipole model with a sum of Gaussians, and use them to blur the irradiance signal in texture space. Since the Gaussians are separable, this allows transforming the expensive $2 \mathrm{D}$ convolutions into a cheaper set of 1D convolutions. This technique enables realtime frame rates, while giving results that are comparable to offline simulations. In follow-up work, additional optimizations are introduced, based on computing a single 2D convolution at 13 jittered sample points, which account for direct reflection and two levels of scattering [HBH09]; unfortunately, 13 samples are not enough for a 2D convolution, which leads to poor results.

Although these techniques provide real-time frame rates, they scale poorly with the number of translucent objects in the scene, since the subsurface-scattering simulation needs to be performed on a per-object basis. To overcome this, Jimenez et al. [JSG09, JG10] propose to translate the simulation from texture to screen space. The diffuse reflection of the translucent object is blurred as a post-processing step employing the sum-of-Gaussians formulation, thereby limiting subsurface scattering computations to the visible parts of the objects. Other techniques operating in screen space include the work of Mertens et al. [MKB* 05], using importance sampling of the BSSRDF, Shah et al. [SKP09], who use a splatting process for integration instead of a gathering step, and Mikkelsen [Mik10], who shows how convolution with a Gaussian can be expressed as a cross bilateral filter. Penner and Borshukov [PB11] pre-integrate the illumination effects of subsurface scattering due to curvature and shadowing into textures, assuming that surface normals can be pre-blurred, and that soft shadows are used. Recent real-time techniques use the diffusion approximation to render optically thick materials [WZT ${ }^{*} 08$ ], including using finite elements and finite differences to support arbitrary, non-locally flat geometry [WWH* 10, LSR $\left.{ }^{*} 13\right]$. In contrast to these works, our technique defers the blurring until the shading has been computed, thus retaining all the geometric detail. Furthermore, it is simpler to implement and yields very high frame rates, suitable for the most challenging real-time scenarios.

\section{Separable Subsurface Scattering}

The diffuse reflectance of a homogeneous translucent material due to subsurface light scattering is characterized by its 2D diffuse reflectance profile $R_{d}(x, y)$, which describes the light reflected around a normally incident pencil beam on the origin of a surface of an infinite half-space [JMLH01]. For a homogeneous material, $R_{d}$ is radially symmetric, and can be characterized by a $1 \mathrm{D}$ diffusion profile $R_{d}(r)$ such

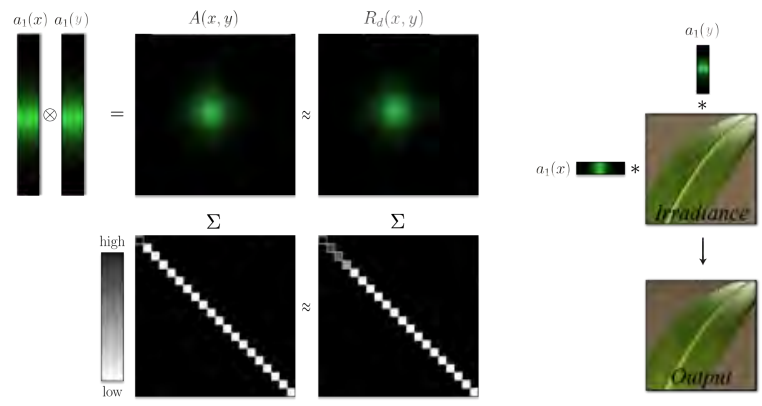

Figure 3: Overview of our approach: based on the low-rank nature of the diffusion kernel $R_{d}(x, y)$, shown by the $\Sigma$ matrix below storing the singular values of the kernel (magnitude in grayscale), we approximate $R_{d}(x, y)$ with $A(x, y)=$ $a_{1}(x) a_{1}(y)$. This simplifies the simulation of subsurface scattering (right) to just two $1 \mathrm{D}$ convolutions per summand with the irradiance signal.

that $R_{d}(x, y)=R_{d}(\|(x, y)\|)^{\dagger}$. It can be used to calculate the radiant exitance $M_{e}(x, y)$ at an arbitrary surface point $(x, y)$ according to:

$$
M_{e}(x, y)=\int_{\mathbb{R}^{2}} E\left(x^{\prime}, y^{\prime}\right) R_{d}\left(x-x^{\prime}, y-y^{\prime}\right) d x^{\prime} d y^{\prime},
$$

where $E(x, y)$ is the irradiance at point $(x, y)$, and both $M_{e}(x, y)$ and $E(x, y)$ are measured in $W^{-2}$. Note that Equation (1) has the form of a $2 \mathrm{D}$ convolution with the $2 \mathrm{D}$ reflectance profile: $M_{e}(x, y)=\left(E * R_{d}\right)(x, y)$.

Approximation of 2D diffusion profiles. For real-time applications, carrying out the 2D convolution in Equation (1) is prohibitively expensive. However, if we can express the profile $R_{d}$ as an approximation $A$ consisting of a sum of separable functions, it is possible to approximate this operation by a sequence of $2 N 1 \mathrm{D}$ convolutions, which exhibit significantly smaller computational complexity:

$$
\begin{gathered}
\left(E * R_{d}\right)(x, y) \approx(E * A)(x, y)=\sum_{i=1}^{N}\left(\left(E * a_{i}\right) * a_{i}\right)(x, y) \\
\text { with } A(x, y)=\sum_{i=1}^{N} a_{i}(x) a_{i}(y),
\end{gathered}
$$

where the approximation $A$ is defined by 1D functions $a_{i}$. From the radial symmetry of $R_{d}$ it follows that the same functions $a_{i}$ can be employed in both coordinate directions. d'Eon et al. [dLE07] observed that zero-mean Gaussians $G$

\footnotetext{
$\dagger$ Note that the radial symmetry of the diffusion assumption follows from assuming a normally incident incoming light, which in general does not hold in real applications. For a description of SSS with directional-dependent diffusion we refer the reader to e.g. [DLR $* 9$, HCJ13, FHK15].
} 
are suitable functions for approximation:

$$
R_{d}(x, y) \approx A_{g}(x, y)=\sum_{i=1}^{N} w_{i} G\left(x, y ; \sigma_{i}\right),
$$

where $\sigma_{i}$ denote the standard deviation of the respective Gaussians. Due to the separability of the Gaussian kernel, the convolution with $A_{g}$ can be realized as $2 N 1 \mathrm{D}$ convolutions. Unfortunately, for the most demanding real-time scenarios these $2 \mathrm{~N}$ convolutions are still too expensive.

In practice, however, we only work with discretized diffusion kernels, which can be interpreted and analyzed as 2D matrices. This allows us to make the observation that most of the energy of typical diffusion kernels is stored in the first few singular values, and in particular in the first one (see Figure 2). This means that the diffusion profile can be approximated with a low-rank separable approximation, and that a single separable kernel can reproduce most of the kernel perceptual qualities, if chosen appropriately. The application of such a separable kernel for rendering is illustrated in Figure 3.

An obvious choice for a discretized separable kernel would be using just the first component of the singular value decomposition (SVD), which - according to the Eckhart-Young theorem [EY36] - gives the best low-rank approximation with respect to the Frobenius norm. Unfortunately, the rank-1 approximation of the kernel using SVD exhibits a rather large energy loss and produces unsatisfactory results even if energy conservation is enforced by normalizing the kernel. This is due to the fact that a pure kernel-space factorization does not take into account that in image-space, some parts of the kernel are more relevant than others. Higher-rank SVD-based approximations (i.e., $N \approx 2-6$ ) converge very rapidly to the original kernel (Figure 4), but the increased computation times make it a less attractive option for real-time applications. In the following we show that, under certain assumptions, even a rank-1 approximation can be used to reconstruct the diffusion kernel with high accuracy (Section 4), and then propose an artist-friendly separable model that allows intuitive editing of the appearance of translucent materials (Section 5).

\section{Pre-integrated Separable Kernel}

Both the sum-of-Gaussians approximation and our SVDbased method produce unsatisfactory results for a single summand, i.e. $N=1$. Due to the non-separability of discretized representations of realistic diffusion profiles, it is not possible to fully reconstruct the effect of their convolution with 2D signals by a single separable kernel. Additionally, separable approximation kernels are in general not radially symmetric, as illustrated by an example in Figure 5. It is, however, possible to completely reproduce a profile's behavior on a special class of signals: assuming that the irradiance is additively separable, i.e., $E(x, y)=E_{1}(x)+E_{2}(y)$ or, equivalently, $\frac{\partial E}{\partial x \partial y}=\frac{\partial E}{\partial y \partial x}=0$, the radiant exitance $M_{e}$ is given as follows (refer to the supplementary material for details):

$$
\begin{aligned}
M_{e}(x, y)= & \iint E\left(x^{\prime}, y^{\prime}\right) R_{d}\left(x-x^{\prime}, y-y^{\prime}\right) d x^{\prime} d y^{\prime} \\
= & \int E_{1}\left(x^{\prime}\right) \underbrace{\int R_{d}\left(x-x^{\prime}, y-y^{\prime}\right) d y^{\prime}}_{a_{p}\left(x-x^{\prime}\right)} d x^{\prime} \\
& \quad+\int E_{2}\left(y^{\prime}\right) \underbrace{\int R_{d}\left(x-x^{\prime}, y-y^{\prime}\right) d x^{\prime}}_{a_{p}\left(y-y^{\prime}\right)} d y^{\prime} \\
= & \iint E\left(x^{\prime}, y^{\prime}\right) \frac{1}{\left\|a_{p}\right\|_{1}} a_{p}\left(x-x^{\prime}\right) a_{p}\left(y-y^{\prime}\right) d x^{\prime} d y^{\prime},
\end{aligned}
$$

where $a_{p}$ denotes the pre-integrated 1D kernel of $R_{d}$ along a coordinate axis. Due to the radial symmetry of $R_{d}$, we have $a_{p}(x)=a_{p}(y)$, where $\left\|a_{p}\right\|_{1}=\left\|R_{d}\right\|_{1}$ by definition. Hence, we define the pre-integrated ${ }^{\ddagger}$ kernel $A_{p}$ of the diffusion profile as:

$$
A_{p}(x, y)=\frac{1}{\left\|R_{d}\right\|_{1}} a_{p}(x) a_{p}(y) .
$$

Note that $A_{p}$ reproduces the exact $2 \mathrm{D}$ convolution with $R_{d}$ in the presence of additively separable irradiance signals, such as straight shadow boundaries of arbitrary orientation or general axis-aligned 1D functions (see Figure 7 for an example of a vertical shadow boundary).

Even for general (i.e., non-additively separable) signals, this approximation yields good results for a wide range of materials and scenarios (see Figure 6 and 13), since most real-world signals $E(x, y)$ can be locally approximated with

\footnotetext{
$\ddagger$ Note that our pre-integrated formulation is fundamentally different from the one proposed by Penner and Borshukov [PB11]: while they pre-integrate the gradients in image-space due to subsurface scattering, we pre-integrate the kernel, which is later applied to simulate SSS.
}

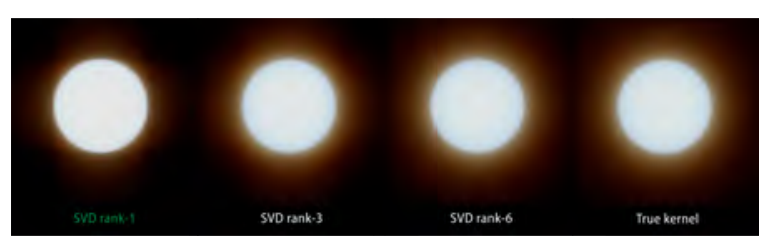

Figure 4: Results of rank- $N$ approximations obtained using the SVD of the discrete diffusion profile for skin, for $N=$ $\{1,3,6\}$. Using the SVD's rank-1 (separable) approximation leads to poor results, since most of the kernel's energy is stored in the center of the kernel. Increasing the rank of the approximation leads to a more faithful approximation of the diffusion kernel, but at the cost of introducing several passes, which makes it inefficient for time-constrained applications. We refer to the supplementary material for more results using the SVD low-rank approximation. 


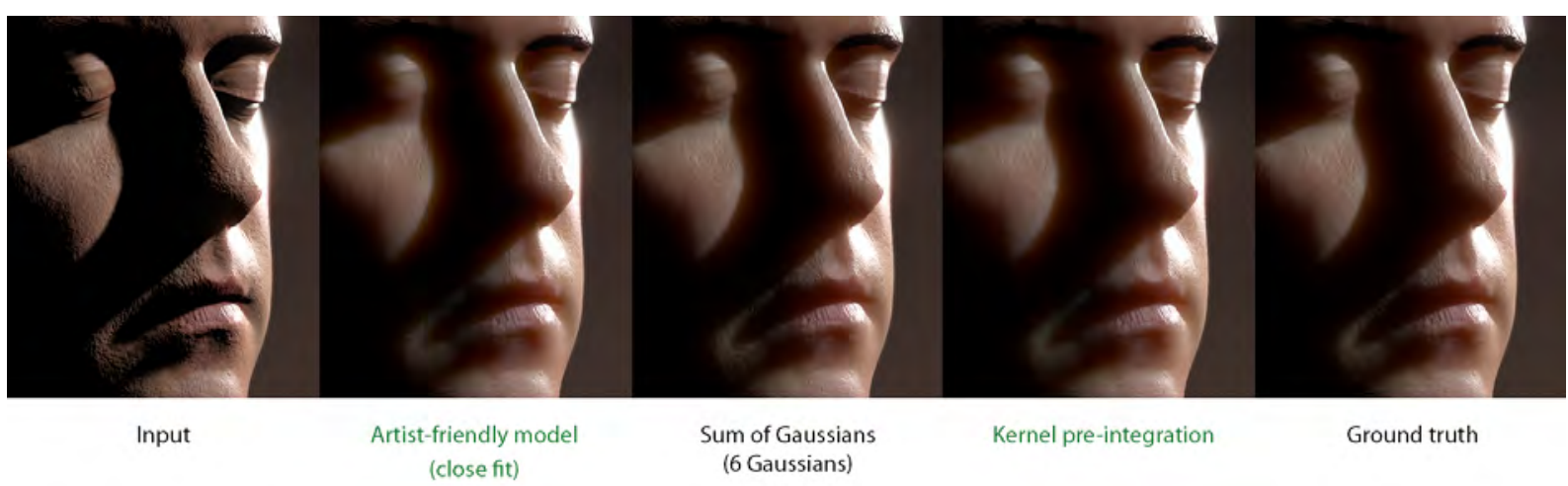

Figure 6: Comparison of the different techniques proposed with a six-Gaussian fit for skin [dLE07, JSG09] and the ground truth (actual 2D kernel). Note that our separable approximations lead to similar quality results with just two 1D convolutions, as opposed to the twelve needed by the sum-of-Gaussians approach.
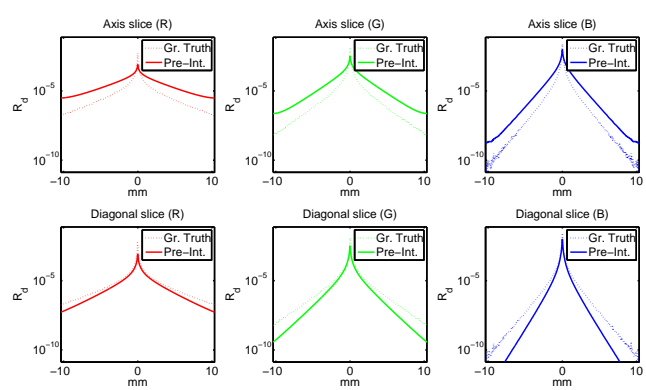

Figure 5: Plot of our pre-integrated kernel compared to the ground truth for human skin, in both the axial and diagonal directions; it can be seen that due to the loss of radial symmetry, our kernel gives different results for the axial and diagonal directions, as opposed to the ground-truth kernel. Note that our method does not try to mimic the kernel to be close to the actual 2D diffusion kernel, but it tries to match the final result of the convolution (Figure 6 and 7). Additional comparisons can be found in the supplementary material.

additively separable functions. However, this formulation offers limited control to an artist and needs to be discretized to be used in practical applications; in the following section we describe a separable, artist-friendly model that overcomes these two limitations.

\section{An Artist-Friendly Separable Model}

Our pre-integrated kernel (Section 4) is able to reconstruct a wide range of materials given its diffusion kernel, which can be obtained from measured data [JMLH01, MES*11] or from simulations. However, in a production environment these profiles might not be the optimal ones, since they might not match the assets (e.g., color of the albedo maps) being captured or computed for different types of skins. To solve

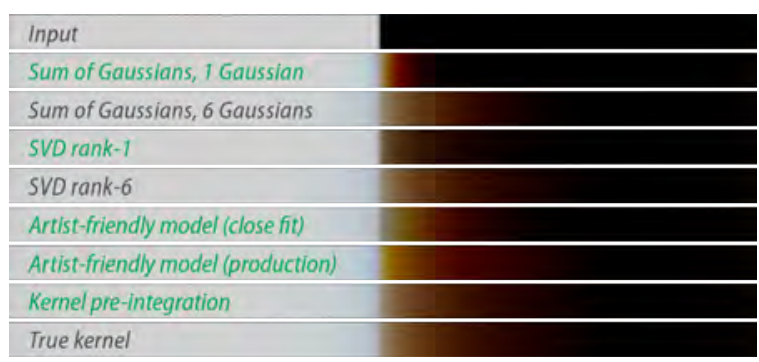

Figure 7: Comparison of the results for our two separable techniques applied to a step-like irradiance: both the sumof-Gaussians [dLE07, JSG09] and a low-rank SVD-based decomposition need several convolutions to match the true kernel. Our proposed pre-integrated kernel, however, is exact for axis-aligned functions, such as this example. Our artistfriendly model provides a rich design space: This is illustrated by two approximations, where for the first (close fit), perceptual similarity to the ground truth was the modeling objective, while the second (production) was tweaked by an artist for production purposes. Note that the image is best viewed in the electronic version of the paper. The style of presentation was inspired by previous work [DI11, HCJ13]. Additional 2D visualizations of the different kernels are provided in the supplementary materials, where we also demonstrate that the radial asymmetry of the SVD-based kernels vanishes rapidly with increasing rank.

this issue, we propose an approach suitable for artistic editing of subsurface scattering, based on physically meaningful parameters.

A general approximation adding separable kernels (see Equation 2) exhibits a vast amount of degrees of freedom if general functions $a_{i}$ are used. Even a sum of $N$ Gaussians (see Equation 3) requires the manipulation of $2 N$ parameters per 

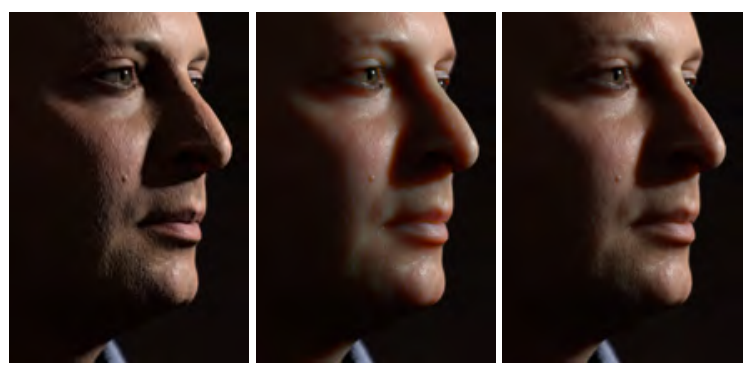

Figure 8: An example of the intuitive editing capabilities of our artist-friendly model. Left: Input irradiance map, without subsurface scattering. Middle: Adjusting the far scattering. Right: Final result after adjusting the near scattering and the balance between the two. Shifting more energy to near scattering allows preserving the bump details.

wavelength by the artist. Moreover, changing the parameters of one Gaussian in the presence of many others may lead to unexpected results. Jimenez et al. [JJG12] proposed ad-hoc transformations to a base profile in a separable approximation to overcome this limitation. However, this model lacked an intuitive mapping to the underlying physics of diffusion. Instead, inspired by previous work allowing modeling and editing of subsurface profiles with simple low-dimensional functions [KKCF13], our approach is based on the more intuitive concept of splitting subsurface scattering into near- and far-range scattering, encoded in two Gaussians. These form the basis of our separable kernel $a_{m}$ as:

$$
\begin{gathered}
a_{m}(x)=w G\left(x, \sigma_{n}\right)+(1-w) G\left(x, \sigma_{f}\right) \\
A_{m}(x, y)=a_{m}(x) a_{m}(y),
\end{gathered}
$$

where $\sigma_{n}$ and $\sigma_{f}$ represent the standard deviation of the near and far scattering Gaussians respectively, and $w$ is the weighting factor. Note that $a_{m}$ represents a mixture of two 1D Gaussians $G(x, \sigma)$, which result in a separable (rank-1) kernel. This is different from the 2D Gaussian mixture approach of d'Eon et al. [dLE07], where two Gaussians would not yield a separable (rank-1) kernel, but a solution of rank two.

Our model takes the benefits of the separable approximation described in Section 3, which is able to match the ground truth under different light configurations (Section 4), while offering a rich design space that allows for intuitive editing of the appearance of translucent materials (Figures 8 and 9(d)), including easy integration in production pipelines, where this appearance control is usually required. Our approximation features another important property: since it is based on Gaussians, it is a continuous parametric representation of the profile; this representation allows computing the diffusion profile analytically at run-time, as will be shown in Section 6 . Moreover, the Appendix introduces an additional guided deviation from the actual diffusion kernel, to generate viable rank-1 approximations that emphasize certain translucency effects.



(a) Jitt $10 \%$

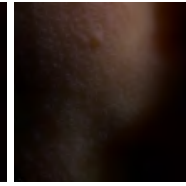

(b) Jitt $30 \%$

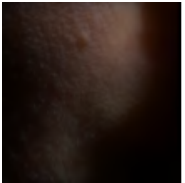

(c) GT

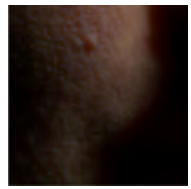

(d) Matched

Figure 9: Although jittering for a radius of $10 \%$ of the kernel size is enough for usual portrait distances and resolutions of 1080p, extreme close-ups may reveal artifacts due to the non-separability of the kernel in these conditions (a). Performing jittering on a radius of $30 \%$ completely removes these artifacts (b), visually matching the result of the ground truth kernel (c). Also note that a kernel designed to match this particular asset using our artist-friendly model (d) shows a more natural look (this image is best viewed in the digital version).
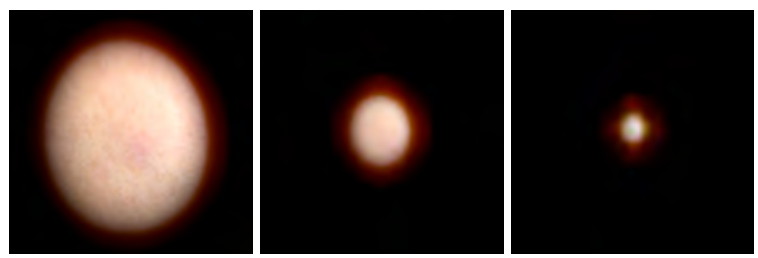

Figure 10: In harsh lighting conditions, progressively smaller features may reveal the fact that the applied separable profiles are not radially symmetric.

\section{Rendering}

Our approximation of the diffusion profile, which is represented as just one separable kernel, can be applied both in texture- and in screen space. For efficiency, we use the screen-space approach of Jimenez et al. [JSG09], including translucency [JWSG10], separating into different buffers the albedo, diffuse and specular components, simulating subsurface transport only in the diffuse layer, and compositing for final rendering. In the following, we highlight additional improvements to the rendering pipeline, resulting in an optimized code of just 16 instructions per sample. We refer the reader to the supplemental material for details.

Jittering. Our separable approximations may lead to some artifacts under high-frequency illumination, as shown in Figure 10. This is because the spatial footprint of the signal becomes smaller than the bandwidth of the kernel, producing an asymmetric star-like pattern. This situation is common also in high-quality, close-up shots, showing for example the pores of skin in detail.

To alleviate this problem, we apply a randomized perpixel rotation of the filtering axes, similar to Huang et al. [HBR $\left.{ }^{*} 11\right]$. Using a randomized rotation, as opposed to other alternatives such as kernel jittering, has two key advan- 


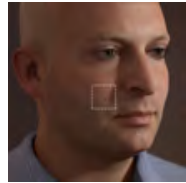

(a)

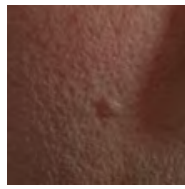

(b) 7 (Uniform)

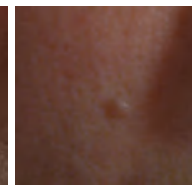

(c) 513 (Unif.)

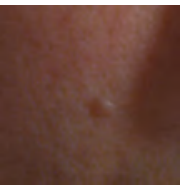

(d) 7 (IS)
Figure 11: The quality of the rendered images, with and without importance sampling of the approximated kernel. (a) Initial image; (b) Uniform sampling with only seven samples leads to obvious aliasing artifacts; (c) Ground-truth created by uniform sampling with 513 samples; (d) Our importance sampling with as low as seven samples.

tages: (i) it breaks the visible cross pattern; and (ii) due to the radial symmetry of the diffusion kernel, it is not necessary to reintegrate the kernel, since distances are preserved. To avoid GPU cache thrashing, we only apply this to samples close to the pixel being evaluated (closer than $10 \%$ of the kernel size in our implementation, although it is dependent on the zoom and the used kernel; for extreme close-ups and kernels modeled with very different Gaussian lobes, a higher range would be needed, as shown in Figure 9). This solves the artifacts in small-scale features such as skin pores, although for higher-scale features, such as the light dot in Figure 10, there may still be visible artifacts (note however that this is a pathological case, not common in real-world applications). Figure 9 demonstrates the effects of randomizing the sample positions. Note that, in addition to masking the artifacts, it also reduces banding problems due to under-sampling.

Kernel footprint and evaluation. For the screen-space method, the size of the convolution kernel is a function of the projected surface area of the pixel [JSG09]. For the continuous approximation (Section 5), we follow similar derivations as Mikkelsen [Mik10]: these allow ( $i)$ applying the kernel accurately in world-space, as opposed to using ad-hoc correction factors [JSG09], and (ii) computing the exact areas for each sample offline, while (iii) still being fast enough for demanding real-time applications. Please refer to the supplementary material (Section E) for low-level details on the implementation.

Importance sampling. To compute the convolution with the 1D functions of the approximation (e.g., $a_{p}$ in Equation 5) during rendering, they need to be discretized. In general these functions exhibit a very uneven energy distribution, so a uniform discretization will either require a high resolution for acceptable quality, which entails a significant performance impact, or result in aliasing when a lower resolution is used. To solve this issue, we use importance sampling on the 1D function by allocating a greater amount of sample points near the center, where most of the energy of the signal is found.

In order to minimize the execution time and the number of memory accesses, we sample all channels at the same posi- tions determined by the dominant channel. In Figure 11, we demonstrate the difference in image quality with importance sampling compared to the ground truth and uniform sampling. In this particular portrait shot under natural lighting, using 7 samples with importance sampling allows a faithful representation of skin subsurface scattering. This importance sampling can also be used to improve the quality of previous methods, such as the sum-of-Gaussians approach [dLE07, JSG09]

\section{Results}

We validate our proposed techniques with a range of different translucent materials. Figures 1 (left), 6, and 12 depict human skin. Figure 7 compares between the different methods also using the skin diffusion profile. Figure 13 features results for soap and marble, while Figure 1 (right) shows ketchup. Additional results are presented in the supplementary material.

We note that the execution time of every technique described in this manuscript is dominated by the convolution computation, so their timings are roughly proportional to the number of convolutions they require (i.e., six Gaussians is approximately six times slower than our separable approximation). Figure 6 compares between our separable approximation, the current state-of-the-art [dLE07, JSG09], and the ground-truth 2D convolution: our pre-integrated kernel gives visually convincing results and can be computed analytically from an arbitrary profile. This enables subsurface scattering to be viable for severely time-constrained real-time applications. Note that in the case of a one-dimensional irradiance signal, such as the one in Figure 7, the pre-integration technique yields the analytic ground truth.

While the artist-controlled approximation allows designing the diffusion arbitrarily, it can also be used to match a specific profile manually; although not as exact as the pre-integrated technique, it still captures key features in a visually convincing way, such as color bleeding into the shadowed region, and it can be seamlessly used in production scenarios. The closefit kernel for human skin (Figures 7, 6 and 9(b,c)) shows how the two-Gaussian artistic model can be used to approximate the ground truth. We again emphasize that this two-Gaussian approach is different from the sum of Gaussians proposed by d'Eon et al. To demonstrate the editing capabilities of our artist-friendly model, we show results using different types of skin, including one actually developed by an artist for improving the results in our particular assets, and another trying to mimic the exact skin diffusion profile (Figure 7), and that we can adapt the appearance of translucency to the goal asset, as seen in Figure 9(d). The parameters for both kernels are included in Table 2.

Our general convolution scheme can be implemented in both texture- and screen space. In the latter, the size of the kernel is a function of the projected surface area in the pixel [JSG09]. As this leads to a favorable fixed-cost property that is sought-after in real-time applications, we believe these 


\begin{tabular}{lccc}
\hline Kernel & $\sigma_{n}[\mathrm{RGB}]$ & $\sigma_{f}[\mathrm{RGB}]$ & $w$ \\
\hline Close fit & {$[0.034,0.029,0.016]$} & {$[1,0.48,0.2]$} & 0.37 \\
Production & {$[0.25,0.08,0.02]$} & {$[1,0.3,0.05]$} & 0.6 \\
\hline
\end{tabular}

Table 2: Parameters for our artist-friendly separable model with two different fits illustrated in our results.

techniques offer a desirable choice for such scenarios. Our method does not require any additional considerations for dynamic objects and moving light sources, and scales well with the area $(0.498,0.348$ and $0.304 \mathrm{~ms}$ at $1080 \mathrm{p}$ on an AMD Radeon HD 7970 for a close-up, middle and long shot respectively). The quality of the simulation can be adjusted by setting the number of samples in the kernel, which gives a trade-off between quality and cost.

Limitations and future work. As discussed previously, trading off radial symmetry for separability might create some artifacts revealing the inherent shape of the kernel in extreme close-up views with high-frequency illumination. While the jittering proposed in Section 6 generally solves this issue for very small-scale details (e.g., skin pores), some artifacts may still remain in some situations (Figure 10). However, we have not experienced this phenomenon in any of the practical cases.

Section 3 shows how the error of our reconstruction is determined by the squared sum of the higher-order singular values. Our method relies on taking advantage of the fact that even though the diffusion profiles are of moderately high rank, the information contained therein is highly structured. Using our separable model, we have been able to match the target profiles well in terms of RMS error.

Since the pre-integrated approximation yields a faithful reconstruction of the diffusion profile with only one separable convolution, a low-rank approximation can be found by approximating the difference between the pre-integrated kernel and the actual diffusion profile. This could lead to better lowrank approximations. In our use cases, a low-rank SVD-based reconstruction already provided sufficient quality for ranks equal or larger than three (see supplementary material for results). This suggests that an SVD-based reconstruction of diffusion kernels might be very useful for simulating efficient high-quality subsurface scattering in off-line or interactive environments.

\section{Conclusions}

We have presented two techniques to generate separable approximations of diffuse reflectance profiles to simulate subsurface scattering for a variety of materials using just two $1 \mathrm{D}$ convolutions. Our separable models yield state-of-the-art results in less than 0.5 millisecond per frame, which makes high-quality subsurface scattering affordable even in the most challenging real-time contexts such as games, where every desired effect may have a budget of tenths of a millisecond.

Using axis-aligned pre-integration, we have presented a high-quality separable approximation that is provably optimal for additively separable irradiance signals. We also proposed an artist-friendly model that allows intuitive artistic control on the appearance of subsurface scattering based on only three parameters, and that allows seamless integration into our separable framework. We have additionally shown that low-rank approximations based on matrix factorization yield higher performance than the sum of Gaussians, which suggests an interesting avenue of future work for efficient subsurface scattering simulation.

Our algorithm works as a post-processing step, which makes it very efficient and simple to integrate in existing rendering pipelines, reducing the complex subsurface light transport [JMLH01] to its barebones (a seven samples blur filter with 16 assembly instructions per sample). Moreover, we have shown how combining importance sampling and jittering strategies allows using only seven samples per pixel in many cases of practical interest.

\section{Acknowledgments}

The authors want to thank the reviewers for their insightful comments; Infinity Realities, in particular Lee Perry-Smith, for his head model and for the Lauren model; the Institute of Creative Technologies at USC, in particular Paul Debevec, for the Ari and Bernardo models; and Bernardo Antoniazzi for letting us use his likeness. Furthermore, we want to thank the Stanford University Computer Graphics Laboratory for the Dragon model, and the following contributors from Blend Swap under CC-BY licence: longrender for the Dish model, metalix for the Green apple model, betomo16 for the Plant model, and PickleJones for the Grapes model. We also thank Felícia Fehér for editing the figures. This research has been partially funded by the European Commission, 7th Framework Programme, through projects GOLEM and VERVE, the Spanish Ministry of Economy and Competitiveness through project LIGHTSLICE, and project TAMA, and the Austrian Science Fund (FWF) through project no. P23700-N23.

\section{References}

[BL03] BorshuKov G., LEWIS J. P.: Realistic human face rendering for 'The Matrix Reloaded'. In ACM SIGGRAPH 2003 Sketches \& Applications (2003). 2

[Cha60] Chandrasekhar S.: Radiative Transfer. Dover Books on Intermediate and Advanced Mathematics. Dover Publications, 1960. 2

[DI11] D'EON E., IRVING G.: A quantized-diffusion model for rendering translucent materials. ACM Trans. Graph. 30, 4 (2011). 2,5

[DJ05] DONNER C., JENSEN H. W.: Light diffusion in multilayered translucent materials. ACM Transactions on Graphics 24, 3 (2005), 1032-1039. 2 

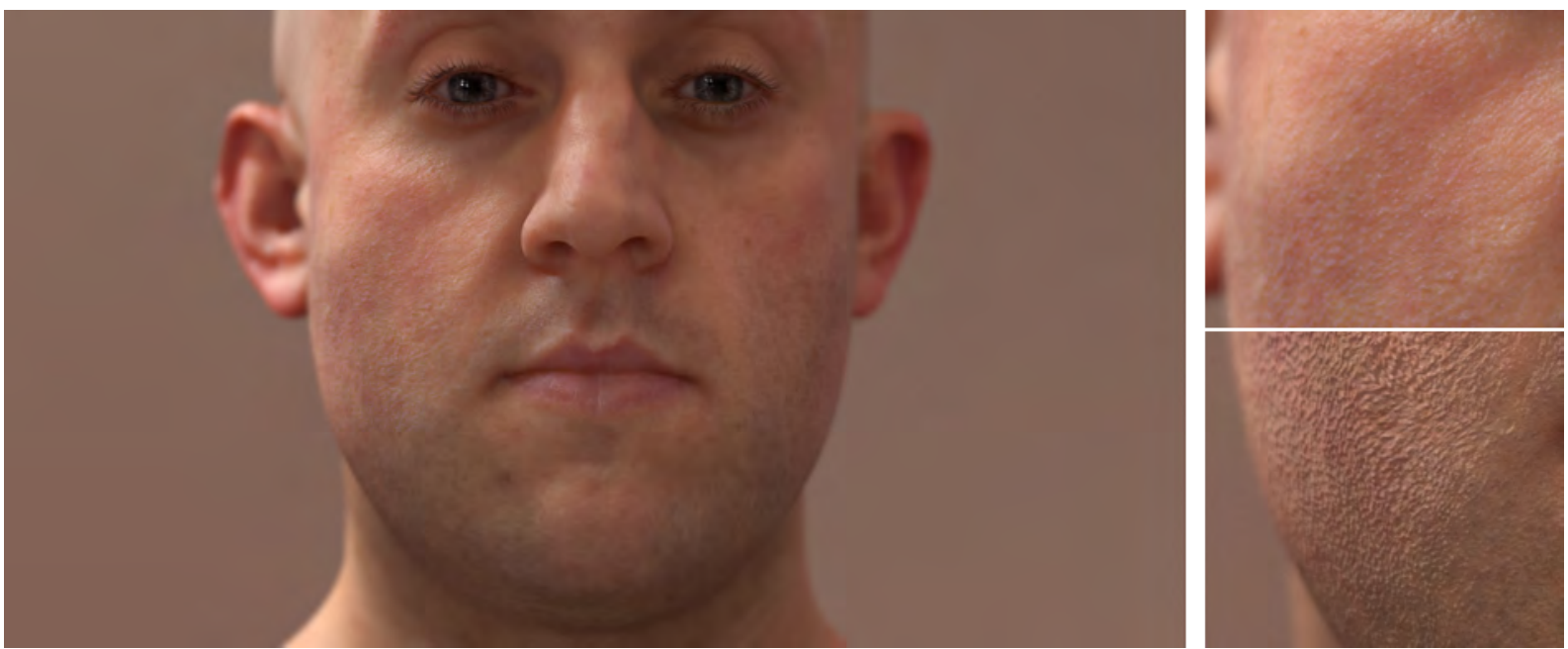

Figure 12: A close-up comparison before (bottom right) and after (top right) applying our separable subsurface-scattering model. Note that without taking into account subsurface light transport, the realism of the image is lost.
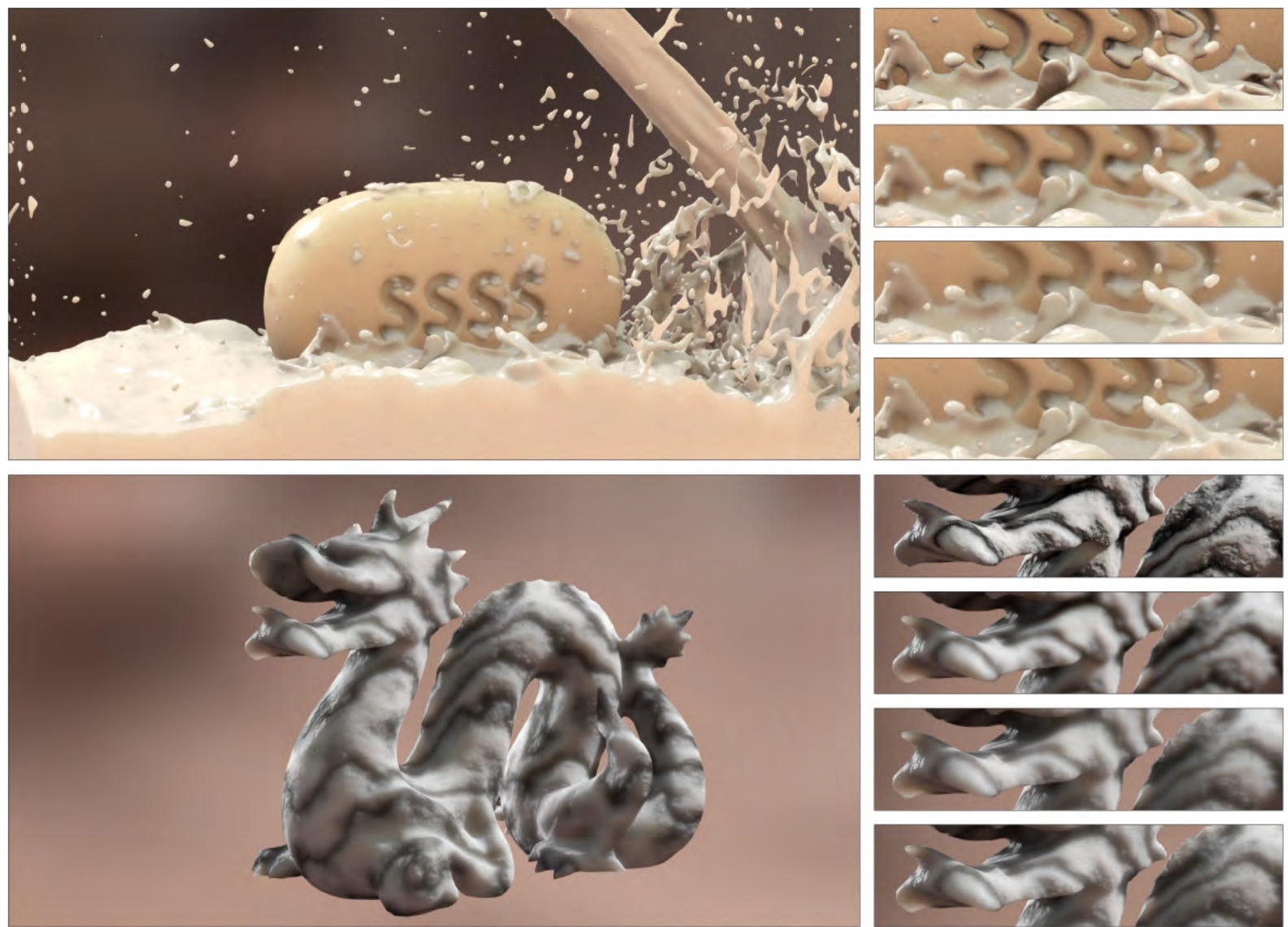

Figure 13: Real-time results for soap and marble. The insets show (from top to bottom) input irradiance, the sum-of-Gaussians approach [dLE07] with 1 Gaussian, our analytic kernel pre-integration technique and the ground truth. Both the sum of Gaussians and ours are run with the same number of convolutions, thereby yielding similar execution times. Our method is able to faithfully capture the effect of subsurface scattering in shadowed regions (soap), and retains intricate texture features (dragon). 
[DJ07] DONNER C., JENSEN H. W.: Rendering translucent materials using photon diffusion. In Proceedings of the Eurographics Symposium on Rendering (2007), pp. 243-252. 2

[dL07] D'EON E., LUEBKE D.: Advanced techniques for realistic real-time skin rendering. In GPU Gems 3, Nguyen H., (Ed.) Addison Wesley, 2007, ch. 14, pp. 293-347. 3

[dLE07] D'EON E., LUEBKE D., ENDERTON E.: Efficient rendering of human skin. In Proceedings of Eurographics Symposium on Rendering (2007), pp. 147-157. 1, 2, 3, 5, 6, 7, 9

[DLR*09] DONNER C., LAWRENCE J., RAMAMOORTHI R., HACHISUKa T., Jensen H. W., NAYAR S.: An empirical bssrdf model. ACM Trans. Graph. 28, 3 (2009). 3

[EY36] ECKART C., YOUNG G.: The approximation of one matrix by another of lower rank. Psychometrika 1, 3 (1936), 211-218. 4

[FHK15] Frisvad J. R., HAChisuka T., KJeldSEN T. K.: Directional dipole model for subsurface scattering. ACM Trans. Graph. To Appear (2015). 3

[HBH09] HABle J., BORShuKOV G., HeJl J.: Fast skin shading. In Shader X7, Engel W., (Ed.). Charles River Media, 2009, ch. 2.4, pp. $161-173.3$

[HBR*11] Huang J., Boubekeur T., Ritschel T., HollänDER M., EISEMANN E.: Separable approximation of ambient occlusion. In Eurographics 2011-Short papers (2011). 6

[HCJ13] Habel R., Christensen P. H., Jarosz W.: Photon beam diffusion: A hybrid monte carlo method for subsurface scattering. Computer Graphics Forum (Proceedings of EGSR 2013) 32, 4 (2013). 2, 3, 5

[JB02] Jensen H. W., BUhler J.: A rapid hierarchical rendering technique for translucent materials. ACM Transactions on Graphics 21, 3 (2002), 576-581. 2

[JG10] Jimenez J., Gutierrez D.: GPU Pro: Advanced Rendering Techniques. AK Peters Ltd., 2010, ch. Screen-Space Subsurface Scattering, pp. 335-351. 2, 3

[JJG12] Jimenez J., Jarabo A., Gutierrez D.: Separable Subsurface Scattering. Tech. rep., Universidad de Zaragoza, 2012.

[JMLH01] JENSEN H., Marschner S., LeVoy M., HANRAHAN P.: A practical model for subsurface light transport. In Proceedings of ACM SIGGRAPH 2001 (2001), pp. 511-518. 2, 3, 5,8

[JSG09] Jimenez J., Sundstedt V., Gutierrez D.: Screenspace perceptual rendering of human skin. ACM Transactions on Applied Perception 6, 4 (2009), 1-15. 1, 2, 3, 5, 6, 7

[JWSG10] Jimenez J., Whelan D., Sundstedt V., GutierREZ D.: Real-time realistic skin translucency. IEEE Computer Graphics and Applications 30, 4 (2010), 32-41. 6

[KF12] Kulla C., FAJARdo M.: Importance sampling techniques for path tracing in participating media. Comp. Graph. Forum 31, 4 (June 2012), 1519-1528. 2

[KKCF13] King A., Kulla C., Conty A., Fajardo M.: Bssrdf importance sampling. In ACM SIGGRAPH 2013 Talks (2013).

[LSR*13] Li D., SUN X., REN Z., Lin S., TONG Y., GuO B., ZHOU K.: Transcut: Interactive rendering of translucent cutouts. Visualization and Computer Graphics, IEEE Transactions on 19, 3 (2013), 484-494. 3

[MES*11] Munoz A., Echevarria J. I., SERon F., LopezMoreno J., GLENCROSS M., GUTIERREZ D : BSSRDF estimation from single images. Computer Graphics Forum 30 (2011), 455-464. 5
[Mik10] Miknelsen M.: Skin Rendering by Pseudo-Separable Cross Bilateral Filtering. Tech. rep., Naughty Dog Inc., August 2010. 3, 7

[MKB*05] Mertens T., Kautz J., BeKaert P., Reeth F. V. SEIDEL H. P.: Efficient rendering of local subsurface scattering. Computer Graphics Forum 24, 1 (2005), 41-50. 3

[PB11] Penner E., Borshukov G.: GPU Pro 2. AK Peters Ltd., 2011, ch. Pre-Integrated Skin Shading, pp. 41-55. 3, 4

[SKP09] Shah M. A., Konttinen J., Pattanaik S.: Imagespace subsurface scattering for interactive rendering of deformable translucent objects. IEEE Computer Graphics and Applications 29 (2009), 66-78. 3

[Sta95] STAM J.: Multiple scattering as a diffusion process. In Eurographics Rendering Workshop (1995), pp. 41-50. 2

[WWH*10] Wang Y., Wang J., Holzschuch N., SUBR K. YONG J.-H., GUO B.: Real-time rendering of heterogeneous translucent objects with arbitrary shapes. In Computer Graphics Forum (2010), vol. 29, Wiley Online Library, pp. 497-506. 3

[WZT*08] WANG J., ZhaO S., Tong X., Lin S., Lin Z., Dong Y., GuO B., SHum H.-Y.: Modeling and rendering of heterogeneous translucent materials using the diffusion equation. $A C M$ Trans. Graph. 27, 1 (Mar. 2008), 9:1-9:18. 3

\section{Appendix A: Guided optimization}

For a guided deviation from the actual diffusion kernel, we additionally present a practical optimization framework to generate viable rank-1 approximations that emphasize the translucency effects of certain features. We aim to find a separable approximation $A_{s}(x, y)=a_{s}(x) a_{s}(y)$ to the diffusion profile $R_{d}(x, y)$, defined by the solution to the minimization problem:

$$
\begin{gathered}
a_{s}=\underset{a}{\operatorname{argmin}} \int_{\mathbb{R}^{2}} \Gamma(x, y)\left(R_{d}(x, y)-a(x) a(y)\right)^{2} d x d y \\
\text { subject to }\left\|R_{d}\right\|_{1}=\|a\|_{1}^{2} .
\end{gathered}
$$

We optimize for minimal $\mathcal{L}_{2}$ distance while still retaining energy conservation via the 1-norm constraint (see Section 3). The guide function $\Gamma(x, y)$ provides the means to select the appropriate length scale of intended artistic effect, and has the form:

$$
\Gamma(x, y ; k)=\left(x^{2}+y^{2}\right)^{k / 2}\left(1-e^{-b x^{2}}\right)\left(1-e^{-b y^{2}}\right),
$$

where $k$ denotes the guide parameter and $b$ gives the suppression term of the center cross region (we use $b=50$ ). Consult the supplementary material for a motivation of this function Varying $k$ between 0 and 4 provides control over the perceived sharpness of the approximation $A_{s} . k \approx 2$ yields the approximation of the actual diffusion kernel, while $k=0$ provides a visually sharper variant (see Figure 7) and $k=4$ a smoother approximation that models the far-range scattering of the diffusion profile more faithfully. This optimization-based approach approximates diffusion kernels while still allowing to emphasize either near or far scattering with the help of a single parameter. This way, the user can adapt the kernel to a given scene and its specific properties, which is not possible by using the single, fixed solution of the pre-integration scheme. 